Pecvnia, 1 (2005), pp. 125-148

\title{
Aplicación de los gráficos de control en el análisis de la calidad textil
}

\author{
Carmen Huerga Castro \\ Pilar Blanco Alonso \\ Julio Abad González
}

\begin{abstract}
El actual nivel de competencia existente en el sector textil hace que las empresas locales deban lograr y mantener un alto nivel de calidad en sus productos. Para conseguirlo, es preciso tomar medidas desde los niveles iniciales del proceso productivo, que es donde hemos centrado nuestra atención. En este trabajo se pretende mostrar la utilidad de las técnicas del Control Estadístico de Procesos en la evaluación de la calidad textil. Concretamente, se diseñan gráficos de control univariantes y multivariantes para vigilar de forma individual y conjunta distintas características de calidad relacionadas con un proceso de hilatura.
\end{abstract}

Palabras clave: Gráficos de control, vigilancia del proceso, calidad textil.
The present competence in textile sector compels local manufacturers to reach and maintain the high level of quality in its products. In order to get it, it is necessary to take measures from the initial levels of the productive process, and that's where we have focused our attention. This paper tries to show the usefulness of the Statistical Process Control techniques in evaluating textile quality. In particular, univariate and multivariate control charts are designed to monitor both individually and jointly the different quality characteristics related to the spinning process.

Key words: Control charts, process monitoring, textile quality. 


\section{INTRODUCCIÓN}

El desarrollo de procedimientos adecuados para el estudio y control de los procesos productivos, es fundamental para mantener un nivel óptimo de calidad en los productos. En este sentido, la Estadística juega un papel importante en todas las actividades empresariales relacionadas con la calidad pues proporciona un amplio abanico de técnicas que se pueden aplicar en el control de la misma.

Aunque la expresión "control de la calidad" puede tener distintos enfoques se puede definir como

el conjunto de las actividades técnicas y administrativas mediante el cual se miden las características de calidad de un producto, se compararan con especificaciones o requisitos y se toman acciones correctivas cuando exista una discrepancia entre el funcionamiento real y el estándar (Navarrete 1998: 18-19).

Generalmente estas características suelen ser medibles, lo que hace posible su evaluación y control. En otras palabras las características de calidad son, en realidad, variables aleatorias y se describen por una determinada distribución de probabilidad. Este hecho, junto con la variabilidad que se presenta en los procesos productivos justifica la necesidad de utilizar la Estadística en el control de la calidad.

Desde este punto de vista la vigilancia de los procesos, en concreto los que se llevan a cabo en el sector textil puede realizarse adecuadamente mediante técnicas estadísticas. Así, en su optimización y mejora resultan útiles las técnicas de diseño de experimentos (Miró et al. 2004). Otras técnicas sencillas, como los gráficos de Pareto, o más avanzadas, como el análisis de la varianza, permitirán establecer distintos comportamientos en máquinas o telares en procesos de hilatura o tejeduría (Clemente Marín et al. 2001).

En este trabajo utilizaremos técnicas de Control Estadístico de Procesos (CEP), construyendo gráficos de control, con el fin de prevenir y detectar los problemas cuando se producen, sin esperar a que el proceso productivo haya concluido.

Hemos tomado como referencia una empresa del sector textil, cuya principal actividad consiste en la fabricación de hilo, si bien dispone también de una planta de tejeduría. Nuestro objetivo es controlar características de calidad relacionadas con el proceso de hilatura mediante 
gráficos de control con el fin de identificar las posibles causas de variación presentes en el proceso.

Por ello, se presenta en la primera parte del trabajo la filosofía general de dichos gráficos de control, y se desarrollan brevemente los utilizados en la aplicación práctica posterior.

En la segunda parte se diseñan, construyen e interpretan gráficos univariantes y multivariantes, a partir de los datos relativos al proceso de hilatura recogidos en el laboratorio de la citada empresa textil.

\section{GRÁFICOS DE CONTROL}

En cualquier proceso de fabricación no siempre se producen los mismos efectos, ya que los elementos que intervienen no siempre funcionan de forma exacta, dando lugar a cierta variabilidad, cuyas causas es preciso investigar. La variabilidad de un proceso puede deberse a causas no asignables y a causas asignables. Las primeras, también llamadas aleatorias son de naturaleza probabilística y forman parte de la variación propia del proceso. Algunas de las razones por las que aparecen estas causas son: variabilidad de la materia prima, variabilidad debida a la maquinaria, distinta eficacia de la mano de obra, etc.

Las causas asignables, dan lugar a variaciones irregulares no predecibles, que hay que eliminar y corregir. Son causas cuya naturaleza no es aleatoria y cuando actúan producen efectos que se pueden determinar con certeza y que persisten hasta que se elimine la causa que los produce. El desgaste de la máquina, herramientas en mal estado, etc. son ejemplos de causas asignables.

Cuando se consigue que en un proceso sólo actúen causas no asignables se dice que está bajo control estadístico. Es en esta tarea donde los gráficos de control constituyen una excelente herramienta, pues permiten decidir cuando hay que intervenir en el proceso para modificar una evolución no deseada.

Un gráfico de control representa la evolución en el tiempo de una característica de calidad medida a partir de una muestra. En términos generales, es una representación gráfica en los ejes cartesianos, donde en el eje horizontal se indica el número de la muestra o el tiempo en que se obtiene, y en el eje vertical se indican los valores observados en las muestras. Se representan tres líneas horizontales: 
- Una línea central (LC), que es la norma de calidad preescrita para el proceso.

- Dos líneas horizontales llamadas límite inferior de control (LIC) y límite superior de control (LSC) que se sitúan por debajo y por encima de la LC.

Estos dos límites constituyen los criterios de decisión para el funcionamiento del proceso, es decir, cuando los puntos correspondientes a las observaciones están dentro de estas líneas se dice que el proceso está bajo control, sin embargo, cuando un punto cae fuera de dichos límites se interpreta como una evidencia de que el proceso está fuera de control. En definitiva, un gráfico de control se puede plantear como un contraste de hipótesis en el sentido siguiente:

$H_{0}$ : El proceso se encuentra bajo control estadístico

$H_{1}$ : El proceso se encuentra fuera de control estadístico

La hipótesis nula se puede referir a la media del proceso, a la dispersión del mismo, o bien a la proporción de unidades defectuosas que origina dicho proceso.

Según Shewhart, la forma genérica de un gráfico de control, desde un punto de vista analítico y desde un punto de vista gráfico, es el siguiente:

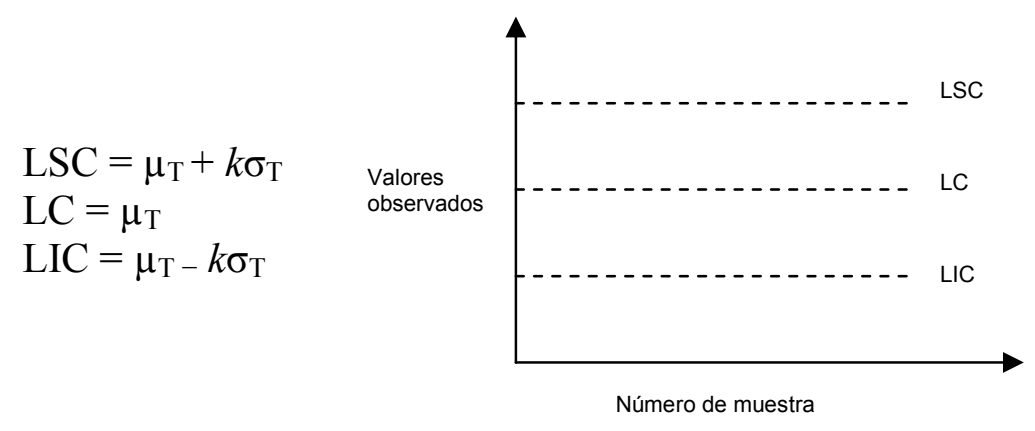

En la expresión anterior:

$T$ es el estadístico muestral que mide la característica de calidad en estudio ( $T$ es el estimador del parámetro del proceso que se desea estimar y controlar). 
$\mu_{\mathrm{T}}$ es la media del estimador.

$\sigma_{\mathrm{T}}$ es la desviación típica del estimador.

$k$ es una constante que indica la distancia de los límites de control a la línea central expresada en unidades de desviación típica.

La posición de los límites de control se determina a partir del valor de la constante $k$, y de la probabilidad de confundirse al tomar una decisión, es decir de cometer un error de tipo I, o un error de tipo II. Normalmente se toma $k$ igual a 3 , en cuyo caso los límites se denominan límites de control 3-sigma, o límites de acción. Cuando la característica de calidad que se está estudiando sigue una distribución normal, la probabilidad de error de tipo I es de $\alpha=0,0027$.

Cuando un punto de la muestra observada se encuentra dentro de los límites de control, no se puede rechazar la hipótesis nula $H_{0}$; por el contrario si está situado fuera de ellos, entonces se rechazará $H_{0}$. Luego los límites superior e inferior de control corresponden respectivamente a los valores superior e inferior de la región de aceptación de la hipótesis nula, para un nivel de significación dado.

Además de los límites 3-sigma, existen otros límites denominados límites de aviso que se encuentran a distancia 2-sigma de la línea central. Como su propio nombre indica sirven de aviso, de modo que si un punto cae entre los límites de acción y los de aviso es posible que el proceso no esté funcionando adecuadamente.

Hay que tener en cuenta, que al igual que en una prueba de hipótesis, es posible que un proceso se juzgue fuera de control cuando realmente este bajo control o viceversa. Las consecuencias de dichos errores son importantes ya que si un proceso se declara fuera de control, cuando en realidad no lo está, se buscarán causas inexistentes; por el contrario si un proceso está fuera de control y se acepta que está en control estadístico, no se logrará el estándar de calidad que se ha fijado.

Otro aspecto a tener en cuenta a la hora de diseñar un gráfico de control es el tamaño de las muestras seleccionadas para llevar a cabo el control del proceso. La decisión sobre el tamaño de muestra se basa en el concepto de "subgrupo racional" introducido por Shewhart (1931; rpt. 1980). Según Shewhart los subgrupos o muestras deben ser tan homogéneos como sea posible para que, si hay presentes causas asignables aparezcan como diferencias entre los subgrupos en lugar de como 
diferencias dentro de los subgrupos. Generalmente se toman muestras de tamaño 4 ó 5 unidades aunque hay ocasiones en las cuales se aconsejan otros tamaños (Calcutt 1995).

Un método para calcular el tamaño de muestra es utilizar la Curva Característica de Operación (CO). Dicha curva es una representación gráfica de la probabilidad de error de tipo II, es decir, la probabilidad de no rechazar la hipótesis nula cuando es falsa, que representa el riesgo $\beta$.

Según la característica que se desea observar, los gráficos de control se agrupan en dos grandes bloques: gráficos de control para variables, cuando la característica sea cuantitativa y gráficos de control para atributos, en caso de ser cualitativa. Si la característica de calidad es una variable, el control del proceso es equivalente a controlar la media y la desviación de la misma.

Existen otros gráficos como los CUSUM y los EWMA que incorporan toda la información suministrada por los puntos observados, acumulando los datos del pasado y del presente. Estos gráficos son una alternativa a los propuestos por Shewhart en los que sólo se utiliza la información derivada de la última muestra.

Dado que en nuestro trabajo estudiaremos variables cuantitativas, parece oportuno exponer brevemente los gráficos utilizados, en concreto los gráficos $\bar{X}-R$, y los gráficos multivariantes, si bien haremos una referencia a los gráficos $\bar{X}-S$.

\subsection{Gráficos $\bar{X}-R$}

Los gráficos $\bar{X}-R$ se utilizan para controlar dos parámetros básicos de un proceso: la media y la dispersión. Para determinar si un proceso está o no bajo control conviene utilizar los dos gráficos conjuntamente. El procedimiento correcto es el siguiente:

$1^{\circ}$ ) Se construye el gráfico de rangos (también denominado grafico de recorridos o gráfico de amplitudes), gráfico $R$, para comprobar si la variación del proceso está bajo control.

$2^{\circ}$ ) Una vez que la dispersión está en control estadístico se construye el gráfico de medias o gráfico $\bar{X}$.

Si los parámetros $\mu$ y $\sigma$ son desconocidos, como ocurre en el problema que nos ocupa, hay que estimarlos a partir de la información 
muestral. Para llegar a las estimaciones definitivas, se realizan los cambios oportunos, se eliminan los posibles puntos que estén fuera de control y se recalculan los límites, hasta conseguir que el proceso esté bajo control.

Estimación de $\mu$ : El valor estimado de $\mu$ se obtiene promediando las medias muestrales disponibles, es decir mediante $\overline{\bar{X}}$.

Si $\bar{X}_{1}, \bar{X}_{2} \ldots \bar{X}_{\mathrm{m}}$ son las medias de las muestras, se toma como estimador de $\mu$ la media de las medias:

$$
\hat{\mu}=\overline{\bar{X}}=\frac{\bar{X}_{1}+\bar{X}_{2}+\ldots+\bar{X}_{\mathrm{m}}}{m}
$$

Se cumple que $E(\overline{\bar{X}})=\mu$, por tanto $\overline{\bar{X}}$ es un estimador insesgado de $\mu$. La línea central del gráfico de control de la media, será el valor $\overline{\bar{X}}$. Para poder calcular el límite superior e inferior necesitamos estimar la desviación típica $\sigma$.

Estimación de $\sigma$ : Existen varios métodos para la estimación de $\sigma$, pero utilizaremos el basado en las amplitudes o rangos de las muestras, definidos como $R=$ Máx $X_{\mathrm{i}}-$ Mín $X_{\mathrm{i}}$.

Para tamaños de muestras pequeñas procedentes de una población normal, la estimación de $\sigma$ que se obtiene utilizando el rango es prácticamente similar al obtenido con el estimador tradicional $S$ (Grant y Leavenworth 1988: 64). Sin embargo, cuando el tamaño muestral es moderadamente grande ( $n>10$ ó 12 ) el método del rango para estimar $\sigma$ pierde eficiencia.

Por consiguiente, para muestras de pequeño tamaño, se define:

$$
\bar{R}=\frac{1}{m} \sum_{i=1}^{m} R_{\mathrm{i}}
$$

siendo $R_{1}, R_{2} \ldots R_{\mathrm{m}}$ los rangos de las $m$ muestras consideradas.

Se comprueba que:

$$
E(\bar{R})=\frac{1}{m} \sum_{i=1}^{m} E\left(R_{\mathrm{i}}\right)=d_{2} \sigma \quad\left(d_{2}\right. \text { en la Tabla 1) }
$$

Por tanto, un estimador de $\sigma$ viene dado por: 


$$
\hat{\sigma}=\frac{\bar{R}}{d_{2}}
$$

Dicho estimador, es un estimador insesgado de $\sigma$ puesto que:

$$
E(\hat{\sigma})=E\left(\frac{\bar{R}}{d_{2}}\right)=\frac{1}{d_{2}} E(\bar{R})=\frac{1}{d_{2}} d_{2} \sigma=\sigma
$$

A partir de los parámetros estimados se calculan los límites de control para los gráficos $\bar{X}$ y los gráficos $R$, tal y como se establece a continuación.

\section{a) Gráficos $\bar{X}$}

Los límites de control del gráfico de la media son:

$$
\overline{\bar{X}} \pm 3 \hat{\sigma}_{\overline{\mathrm{X}}}=\overline{\bar{X}} \pm 3 \frac{\hat{\sigma}}{\sqrt{n}}=\overline{\bar{X}} \pm \frac{3}{\sqrt{n}} \frac{\bar{R}}{d_{2}}=\overline{\bar{X}} \pm A_{2} \bar{R}
$$

siendo: $A_{2}=\frac{3}{\sqrt{n} d_{2}}$

Los valores de la constante $A_{2}$ se encuentran tabulados para distintos valores de $n$ y se recogen en la Tabla 1.

Por tanto las líneas de control del gráfico se representan:

$$
\begin{gathered}
L S C=\overline{\bar{X}}+A_{2} \bar{R} \\
L C=\overline{\bar{X}} \\
L I C=\overline{\bar{X}}-A_{2} \bar{R}
\end{gathered}
$$

\section{b) Gráficos $R$}

Para construir el gráfico $R$, representaremos gráficamente los rangos de las sucesivas muestras, siguiendo la filosofía general del diseño de los gráficos de control.

La media de $R$ se estima por: $\hat{\mu}_{R}=\bar{R}$ y $\sigma_{R}$ por:

$$
\hat{\sigma}_{R}=d_{3} \frac{\bar{R}}{d_{2}}
$$

Luego los límites de control 3-sigma son: 


$$
\bar{R} \pm 3 \hat{\sigma}_{R}=\bar{R} \pm 3 d_{3} \frac{\bar{R}}{d_{2}}=\left(1 \pm 3 \frac{d_{3}}{d_{2}}\right) \bar{R}
$$

siendo: $D_{4}=1+3 \frac{d_{3}}{d_{2}} \quad,, \quad D_{3}=1-3 \frac{d_{3}}{d_{2}}$

Las constantes $D_{3}$ y $D_{4}$ están tabuladas en la Tabla 1. Cuando $n \leq 6$, la constante $D_{3}$ es negativa. Por ese motivo en la tabla aparece $D_{3}=0$ y el límite inferior de control sería $L I C=D_{3} \bar{R}=0$. En estos casos el gráfico de los rangos no tiene límite inferior de control. En general, el gráfico de control consta de la línea central y los correspondientes límites inferior y superior:

$$
\begin{gathered}
L S C=D_{4} \bar{R} \\
L C=\bar{R} \\
L I C=D_{3} \bar{R}
\end{gathered}
$$

\subsection{Gráficos $\bar{X}-S$}

El funcionamiento de estos gráficos es similar al de los gráficos $\bar{X}-R$ pero cambia el procedimiento para estimar la dispersión. Su utilización se aconseja cuando el tamaño de muestra sea superior a 10. Si se toman $m$ muestras, para cada una se calcula $S$ :

$$
S=\left[\frac{1}{n-1} \sum_{i=1}^{n}\left(X_{\mathrm{i}}-\bar{X}\right)^{2}\right]^{\frac{1}{2}}
$$

Un estimador insesgado de $\sigma$ es: $\quad \hat{\sigma}=\frac{\bar{S}}{c_{4}}$

siendo: $\quad \bar{S}=\frac{1}{m} \sum_{i=1}^{m} S_{\mathrm{i}}$

La media del proceso se sigue estimando mediante $\overline{\bar{X}}$, y siguiendo un razonamiento similar a los anteriores se obtienen las siguientes expresiones para el gráfico de control $\bar{X}-S$ : 


$$
\begin{aligned}
& \text { Gráfico } \bar{X} \\
& L S C=\overline{\bar{X}}+A_{3} \bar{S} \\
& L C=\overline{\bar{X}} \\
& L I C=\overline{\bar{X}}-A_{3} \bar{S}
\end{aligned}
$$

Gráfico $S$

$$
\begin{aligned}
& L S C=B_{4} \bar{S} \\
& L C=\bar{S} \\
& L I C=B_{3} \bar{S}
\end{aligned}
$$

Tabla 1. Constantes para determinar los límites de control de los gráficos $\bar{X}-R$ y $\bar{X}-S$

\begin{tabular}{||l|l||l|l|l|l|l|l|l|l|l||}
\hline \hline & $n$ & 2 & 3 & 4 & 5 & 6 & 7 & 8 & 9 & 10 \\
\hline \hline \multirow{5}{*}{$\bar{X}-R$} & $A_{2}$ & 1,880 & 1,023 & 0,729 & 0,577 & 0,483 & 0,419 & 0,373 & 0,337 & 0,308 \\
\cline { 2 - 11 } & $d_{2}$ & 1,128 & 1,693 & 2,059 & 2,326 & 2,534 & 2,704 & 2,847 & 2,970 & 3,078 \\
\cline { 2 - 11 } & $D_{3}$ & 0 & 0 & 0 & 0 & 0 & 0,076 & 0,136 & 0,184 & 0,223 \\
\cline { 2 - 11 } & $D_{4}$ & 3,267 & 2,574 & 2,282 & 2,114 & 2,004 & 1,924 & 1,864 & 1,816 & 1,777 \\
\hline \hline & $A_{3}$ & 2,659 & 1,954 & 1,628 & 1,427 & 1,287 & 1,1182 & 1,099 & 1,032 & 0,975 \\
\cline { 2 - 10 } & $c_{4}$ & 0,7979 & 0,8862 & 0,9213 & 0,94 & 0,9515 & 0,9594 & 0,965 & 0,9693 & 0,9727 \\
\cline { 2 - 10 } & $B_{3}$ & 0 & 0 & 0 & 0 & 0,03 & 0,118 & 0,185 & 0,239 & 0,284 \\
\cline { 2 - 10 } & $B_{4}$ & 3,267 & 2,568 & 2,266 & 2,089 & 1,97 & 1,882 & 1,815 & 1,761 & 1,716 \\
\hline
\end{tabular}

\subsection{Gráficos Multivariantes}

Los gráficos anteriores estudian sólo una característica de calidad, pero es posible que en un proceso de producción sea necesario vigilar varias características simultáneamente, en cuyo caso estamos ante un control multivariante. Una primera posibilidad sería estudiar cada característica de calidad por separado, es decir aplicar gráficos de control para cada variable que se desea controlar. Así, un proceso se juzgaría bajo control cuando cada uno de los gráficos individuales lo estuviera; sin embargo, no es posible controlar las interacciones entre las diversas variables ni la variación a lo largo de tiempo, pues no se tiene en cuenta la información que aportan las covarianzas y su estabilidad. Un gráfico alternativo es el gráfico $T^{2}$ de Hotelling.

Gráfico de control $T^{2}$ de Hotelling

El gráfico $T^{2}$ de Hotelling es una extensión al caso multivariante del gráfico de control Shewart univariante. Utiliza un estadístico que combina la información para la dispersión y la media de las variables que se están analizando. 
Supongamos que tenemos dos características de calidad $X_{1}$ y $X_{2}$, que se distribuyen conjuntamente según la distribución normal bivariante: $\left(X_{1}, X_{2}\right) \rightarrow N(\mu, \Sigma)$ siendo $\mu$ el vector de medias $\mu=\left(\mu_{1}, \mu_{2}\right)$ y $\sum$ la matriz de varianzas y covarianzas.

A partir de muestras preliminares, se estima el vector de medias con el vector $\overline{\bar{X}}=\left(\overline{\bar{X}}_{1}, \overline{\bar{X}}_{2}\right)$ y la matriz de covarianzas mediante la matriz:

El estadístico:

$$
S=\left(\begin{array}{ll}
S_{1}^{2} & S_{12} \\
S_{21} & S_{2}^{2}
\end{array}\right)
$$

$$
T^{2}=\frac{n}{S_{1}^{2} S_{2}^{2}-S_{12}^{2}}\left[S_{2}^{2}\left(\bar{X}_{1}-\overline{\bar{X}}_{1}\right)^{2}+S_{1}^{2}\left(\bar{X}_{2}-\overline{\bar{X}}_{2}\right)^{2}-2 S_{12}\left(\bar{X}_{1}-\overline{\bar{X}}_{1}\right)\left(\bar{X}_{2}-\overline{\bar{X}}_{2}\right)\right]
$$

sigue una distribución $T^{2}$ de Hotelling con 2, $n-1$ grados de libertad.

Si el valor experimental del estadístico $T^{2}$ es mayor que el valor teórico $T_{\alpha, 2, n-1}^{2}$, es decir, si $T^{2}>T_{\alpha, 2, n-1}^{2}$, entonces al menos una de las dos características de calidad está fuera de control, en consecuencia se rechazará la hipótesis de proceso bajo control. En caso contrario se aceptará la hipótesis.

Gráficamente, si $\bar{X}_{1}$ y $\bar{X}_{2}$ son independientes, es decir, $S_{12}=0$, la ecuación [1] define una elipse centrada en $\left(\overline{\bar{X}}_{1}, \bar{X}_{2}\right)$, con los ejes principales paralelos a los ejes $\bar{X}_{1}$ y $\bar{X}_{2}$. Si las dos características de calidad son dependientes, entonces $S_{12} \neq 0$, lo que hace que los ejes principales de la elipse de control no sean paralelos a los ejes $\bar{X}_{1}$ y $\bar{X}_{2}$, sino que presenten cierta inclinación.

Existen dos desventajas asociadas a la representación mediante la elipse de control:

1.- Se pierde la secuencia en el tiempo de los puntos representados.

2.- Resulta difícil construir una elipse para más de dos características de calidad.

Para evitar estos problemas, el gráfico de control utilizado consiste en representar en los ejes cartesianos los puntos $T^{2}$ calculados a partir de la ecuación [1] para cada muestra, y una línea horizontal en 
$T_{\alpha, 2, n-1}^{2}$ que será límite superior de control. Esta gráfica se llama normalmente diagrama de control $T^{2}$ de Hotelling.

Este método se podría extender al caso de $p$ características de calidad, Montgomery (1991: 322).

\section{EVALUACIÓN DE LA CALIDAD PARA CARACTERÍSTICAS TEXTILES. METODOLOGÍA}

El planteamiento expuesto anteriormente se aplica ahora a la monitorización y vigilancia de determinadas características de calidad de interés en el sector textil. Se ilustra la construcción y uso de dichos gráficos tomando como referencia datos reales obtenidos en el laboratorio de una fabrica textil en relación al proceso de hilatura.

La hilatura es una secuencia de operaciones que transforman las fibras de algodón en rama, en un hilo adecuado para varios productos finales. Nos hemos centrado en el estudio de hilatura convencional de algodón $100 \%$.

El proceso de fabricación incluye una serie de operaciones a partir del algodón que llega a fábrica. Comienza transformando las capas compactas de algodón en copos ligeros y esponjosos. A continuación se realiza el cardado y peinado removiendo sucesivamente los copos, abriéndolos y separándolos para formar una cuerda llamada "torzal". Los pasos siguientes son el estirado y mechado, en los cuales se estiran las fibras del torzal reduciéndolas hasta un valor adecuado.

En la última fase, llamada hilatura, se hila la mecha estirándola hasta el tamaño o número de hilo deseado. Es en esta fase donde hemos centrado la atención estudiando dos características:

- Título Absoluto: Diámetro del hilo.

- Pilosidad: Longitud de la vellosidad del hilo.

\subsection{Diseño y construcción de gráficos de control}

Para elegir el gráfico de control adecuado, es importante conocer, además del tipo de variable que se estudia, la forma en que se recogen los datos para su análisis posterior y el estándar de calidad que se pretende conseguir. 
Diseño de los gráficos de control:

- En el caso que nos ocupa, de la fabricación de cada máquina se van tomando ovillos para realizar las pruebas de calidad en el laboratorio. Los datos utilizados corresponden al primer semestre de 2004 y se refieren a una de las máquinas con las que cuenta la empresa. Dicha máquina está programada para fabricar hilo de título 18. Los datos aparecen agrupados en muestras de tamaño ocho (se recogen ocho ovillos de hilo a intervalos de tiempo regulares).

- Las características que se analizan son de tipo cuantitativo, y el objetivo es comprobar si la media y la dispersión se encuentran en control estadístico, por ello utilizaremos gráficos $\bar{X}-R$. La elección de estos gráficos obedece, no sólo al tipo de característica estudiada, sino también al tamaño de la muestra que, como ya hemos apuntado, es inferior a 10 unidades. No obstante también se presentan los límites obtenidos utilizando gráficos $\bar{X}-S$.

- Un paso previo a la construcción de un gráfico de control es comprobar que la distribución de la característica de calidad es normal, si bien esta hipótesis afecta más a los gráficos $R$ que a los gráficos $\bar{X}$, puesto que estos últimos, en virtud del teorema central del límite, son más robustos frente a la hipótesis de normalidad.

En la tabla 2 aparecen los resultados obtenidos al aplicar el test de Kolmogorov-Smirnow con la corrección de Lilliefors'.

Tabla 2. Test de normalidad para las características de calidad de la hilatura

\begin{tabular}{|l|c|c|}
\cline { 2 - 3 } \multicolumn{1}{c|}{} & \multicolumn{2}{c|}{ Kolmogorov-Smirnov (Lilliefors) } \\
\cline { 2 - 3 } \multicolumn{1}{c|}{} & Estadístico & $\mathrm{p}$-valor \\
\hline Título absoluto & 0,061 & $>0,150$ \\
\hline Pilosidad & 0,049 & $>0.150$ \\
\hline
\end{tabular}

1 El test de Lilliefors es una modificación del test de Kolmogorov-Smirnov que se debe utilizar cuando no se especifican los parámetros media y varianza de la distribución normal con la que se pretende comparar. 
Figura 1. Gráficos de probabilidad normal para las características de calidad
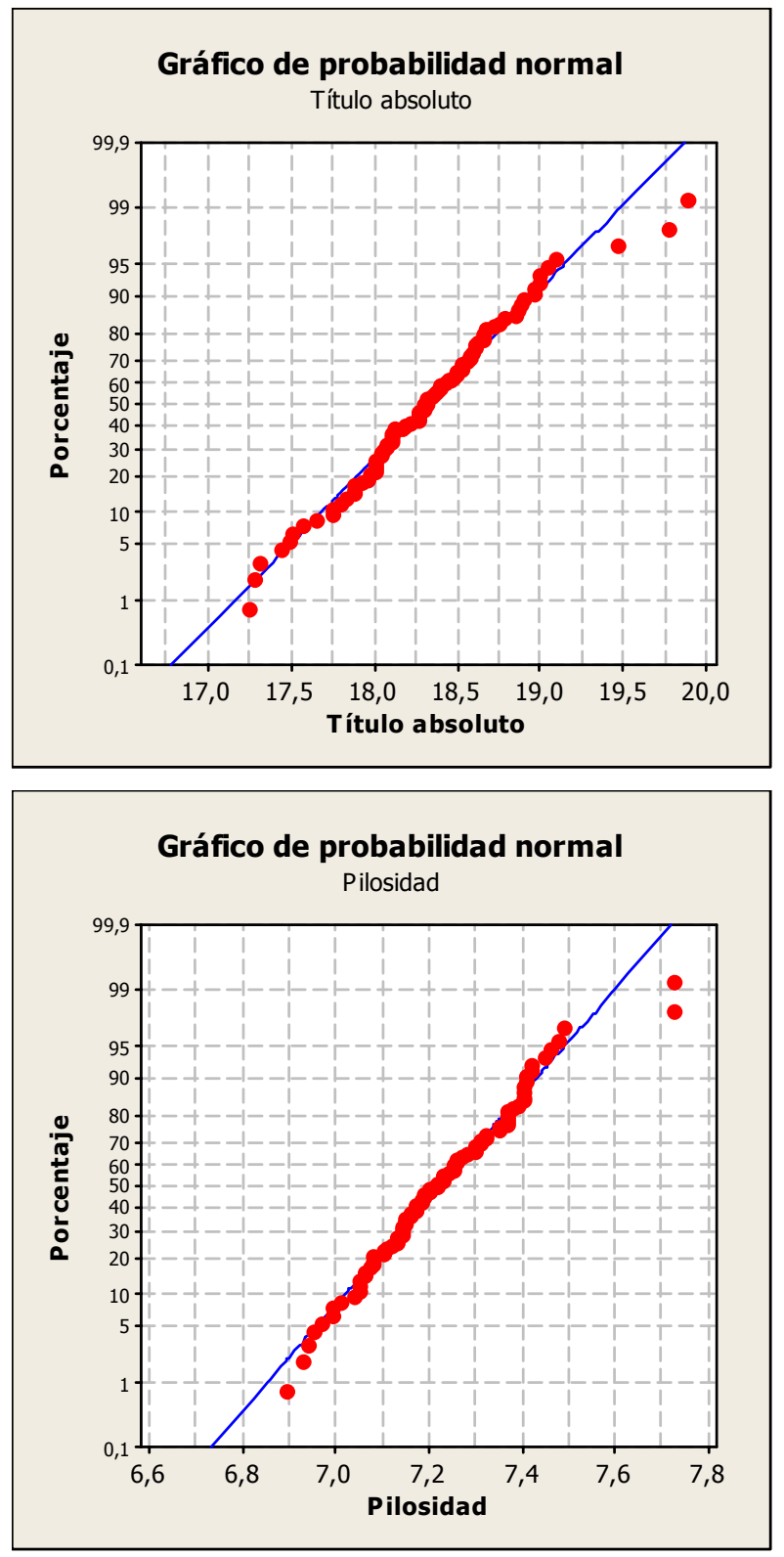

Tomando como referencia el test de Lillierfors, se puede admitir la normalidad de las dos características estudiadas, pues el valor $p$ correspondiente al estadístico es siempre superior a $\alpha=0,05$. La Figura 1 ratifica dicha afirmación, mostrando los gráficos de normalidad de cada característica. 
Construcción de gráficos:

Para construir un gráfico de control $\bar{X}-R$, se debe estimar la media y la dispersión para cada una de las características de calidad señaladas. A modo de ejemplo se detalla el procedimiento para una de las características, en concreto para "Título absoluto".

- Estimación de la media de la característica "Título absoluto"

Para cada una de las 11 muestras de tamaño 8 se calcula su media y después se promedian:

$$
\overline{\bar{X}}=\frac{\bar{X}_{1}+\bar{X}_{2}+\ldots+\bar{X}_{11}}{11}=18,322
$$

Así pues $\hat{\mu}=18,322$.

- Estimación de la dispersión de la característica "Titulo absoluto"

Para cada muestra se obtiene el rango $\left(R=\right.$ Máx $X_{\mathrm{i}}-$ Mín $\left.X_{\mathrm{i}}\right)$ y posteriormente se calcula la media de los mismos.

$$
\begin{gathered}
\bar{R}=\frac{1}{11} \sum_{i=1}^{11} R_{\mathrm{i}}=1,34818 \\
\hat{\sigma}=\frac{\bar{R}}{d_{2}}=\frac{1,34818}{2,847}=0,4735
\end{gathered}
$$

Los límites del gráfico para controlar la media del proceso son los siguientes:

$$
\begin{gathered}
L S C=\overline{\bar{X}}+A_{2} \bar{R}=18,322+0,373 \cdot 1,34818=18,822 \\
L C=\overline{\bar{X}}=18,322 \\
L I C=\overline{\bar{X}}-A_{2} \bar{R}=18,322-0,373 \cdot 1,34818=17,822
\end{gathered}
$$

La línea central y los límites de control para el gráfico de rangos, que es el que nos permite controlar la variabilidad del proceso son:

$$
\begin{gathered}
L S C=D_{4} \bar{R}=1,864 \cdot 1,34818=2,50 \\
L C=\bar{R}=1,342 \\
L I C=D_{3} \bar{R}=0,136 \cdot 1,34818=0,183
\end{gathered}
$$


La representación conjunta se muestra en la Figura 2.

Figura 2. Gráficos $\bar{X}-R$ "Título absoluto"

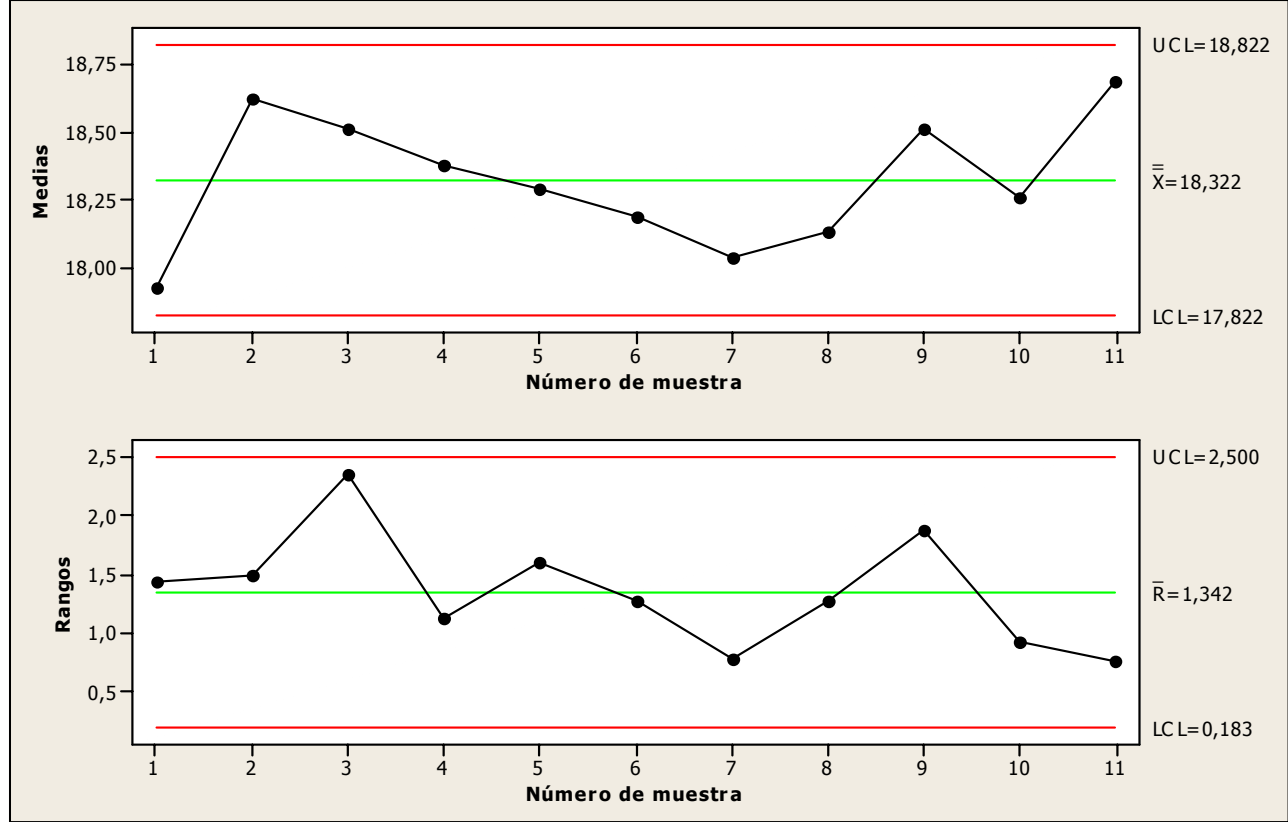

Observando el gráfico de medias se comprueba que todos los puntos están situados dentro de los límites de control, apreciándose una tendencia decreciente desde la muestra número 2 hasta la 7 . No obstante, al tratar de identificar posibles patrones no aleatorios, hemos tomado como referencia los ocho criterios que implementa el paquete estadístico MINITAB ${ }^{2}$, pero no se aprecia ninguno de ellos.

Nos ha parecido oportuno establecer límites más estrechos, en concreto, límites de aviso 2-sigma. En la Figura 3 se muestran los gráficos de control para el "Título absoluto" con límites 2-sigma y 3-sigma. Tanto en el gráfico $R$ como en el gráfico $\bar{X}$ se observa un punto situado en la banda comprendida entre dichos límites, lo que puede sugerir la existencia de alguna incidencia en el proceso. Un estudio más detallado permitió concluir que no existía ninguna causa asignable y se decidió tomar los límites obtenidos como definitivos. En consecuencia se podrán 
utilizar para controlar la producción futura de la máquina de referencia, no sirviendo estos límites para una máquina distinta.

Figura 3. Gráficos $\bar{X}-R$ "Título absoluto" con límites 2-sigma

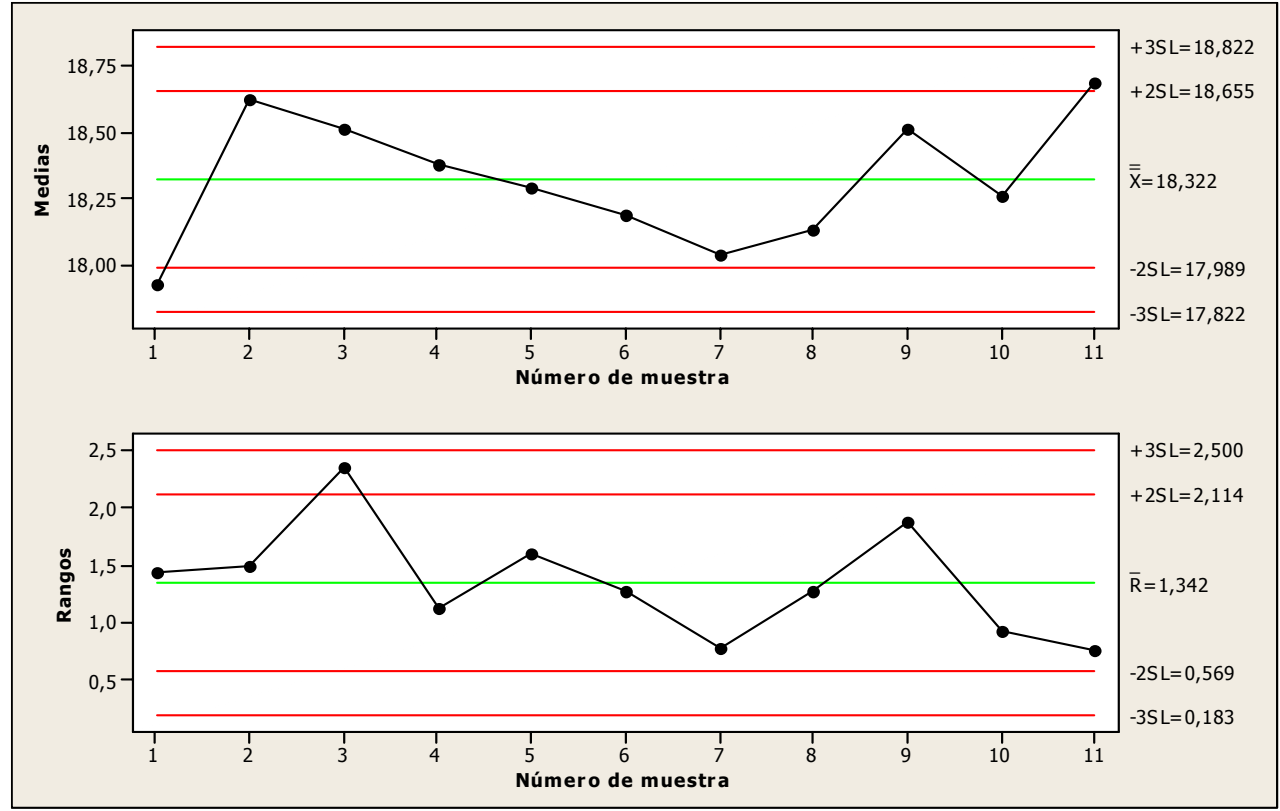

Se han implementado los gráficos $\bar{X}-R$, por ser los más adecuados para el tamaño de muestra 8 . No obstante comparamos los límites calculados con los que se obtendrían de haber utilizado los gráficos $\bar{X}-S$. Hay que señalar que las estimaciones de $\sigma$ calculadas a partir de $S$ y a partir de $R$ son prácticamente idénticas.

Su valor se obtiene mediante la expresión:

$$
\hat{\sigma}=\frac{\bar{S}}{c_{4}}=\frac{0,4547}{0,9650}=0,4712
$$

siendo: $\quad \bar{S}=\frac{1}{m} \sum_{i=1}^{m} S_{\mathrm{i}}$

y $S_{\mathrm{i}}$ la desviación de cada muestra.

En la Tabla 3 se recoge una comparación de los límites correspondientes a los gráficos $R$ y $S$. 
Tabla 3. Límites de los gráficos de control para la dispersión

\begin{tabular}{|l|l|c|}
\cline { 2 - 3 } \multicolumn{1}{c|}{} & Gráfico $R$ & Gráfico $S$ \\
\hline LSC & 2,5 & 0,8253 \\
\hline LC & 1,342 & 0,4547 \\
\hline LIC & 0,183 & 0,0842 \\
\hline
\end{tabular}

También en el estudio de la característica "Pilosidad", que mide la longitud de pelusa del hilo, se han utilizado los gráficos $\bar{X}-R$. El gráfico de los rangos se encuentra en control estadístico, pero en el gráfico de medias aparece un punto situado fuera de los límites de control (Figura 4), por tanto hay que eliminar la muestra correspondiente a esta media y recalcular los límites.

Figura 4. Gráficos $\bar{X}-R$. "Pilosidad"

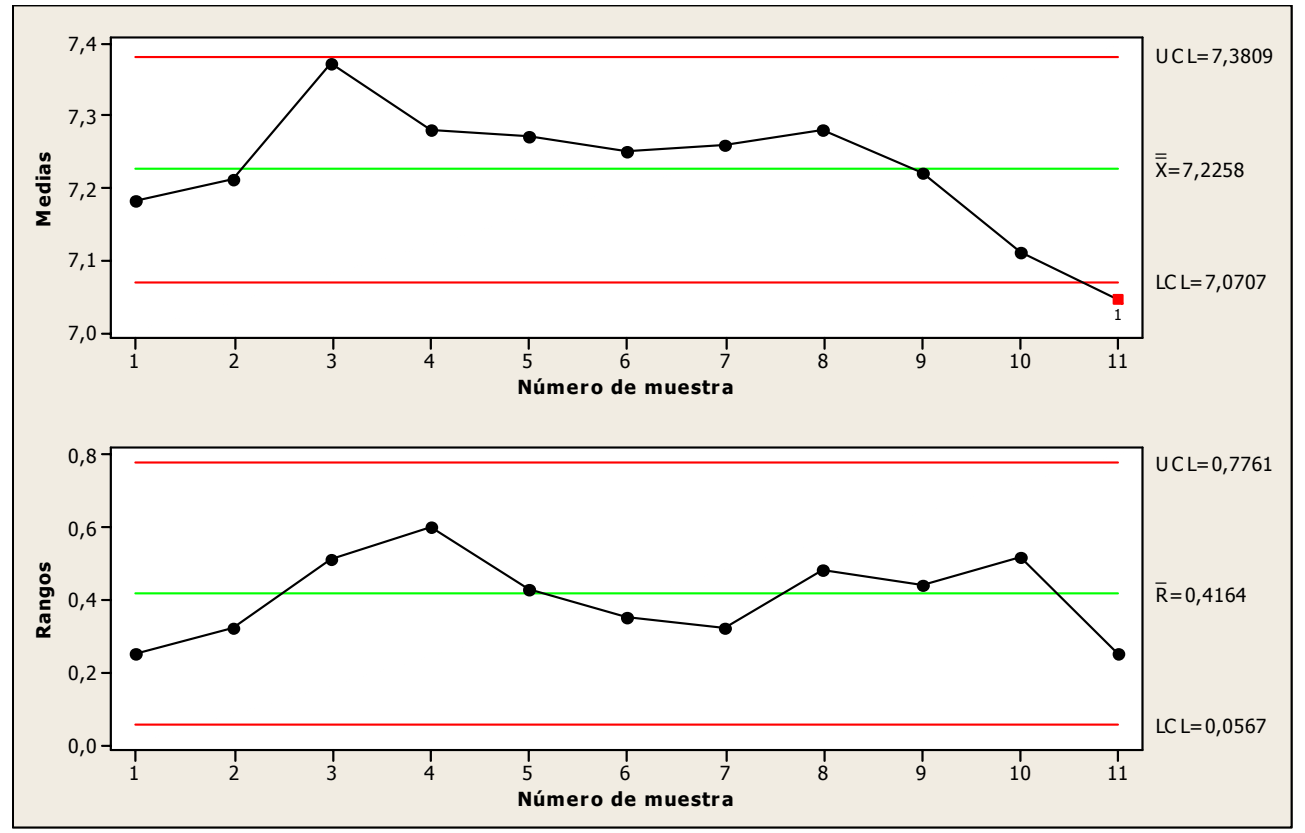

El gráfico resultante aparece en la Figura 5 donde se observa que todas las medias están en control estadístico. Se detecta una clara tendencia decreciente, que ya se manifestaba en el gráfico de la Figura 4. Por tanto el gráfico que muestra el proceso bajo control (Figura 5) debería tomarse con reservas si se utiliza para controlar la producción futura. No obstante si el objetivo es reducir la vellosidad, es posible que se haya realizado algún ajuste en la máquina que esté provocando dicha situación. 
Figura 5. Gráficos $\bar{X}-R$. "Pilosidad" (eliminando una muestra)

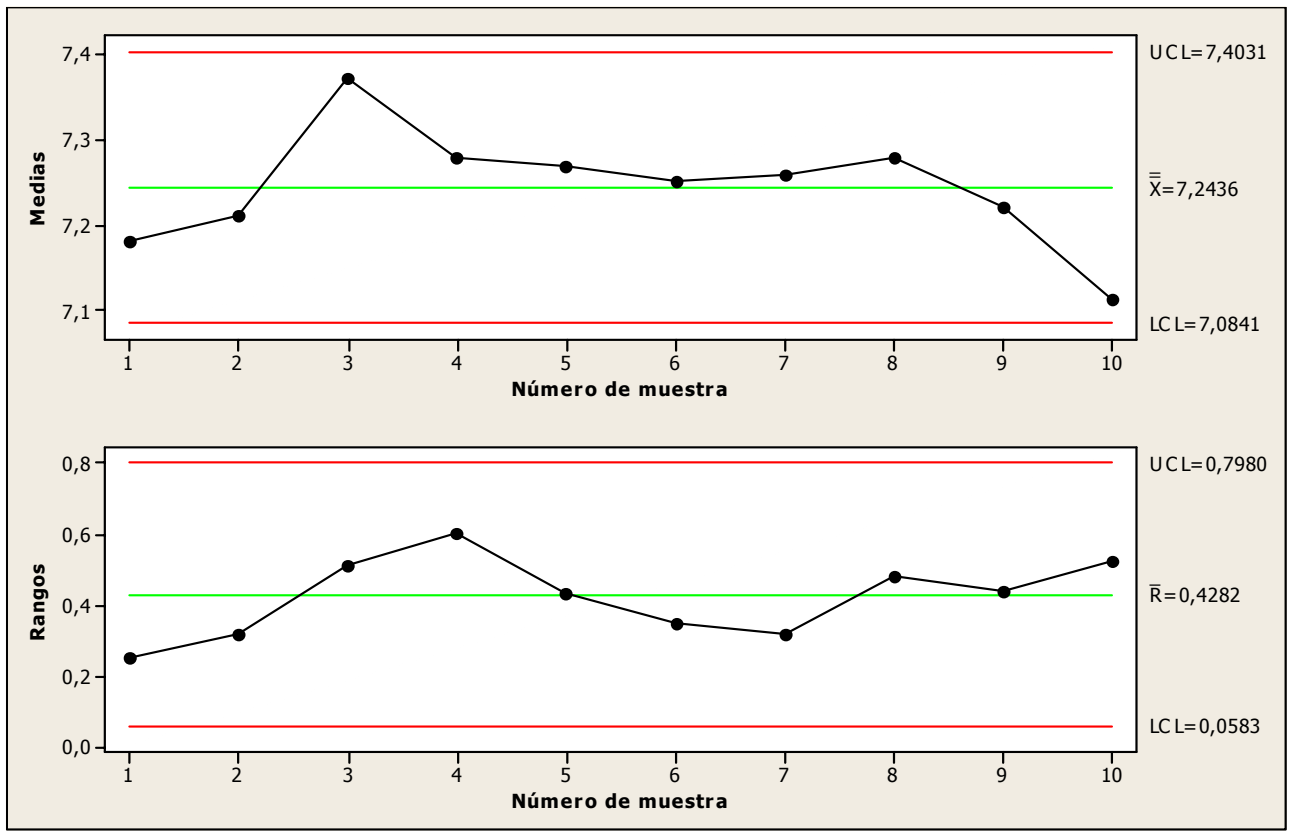

Para completar el estudio hemos diseñado gráficos multivariantes $T^{2}$ de Hotelling con el fin de controlar conjuntamente las dos características "Título absoluto" y "Pilosidad", es decir para analizar a la vez el grosor del hilo y la longitud de la pelusa del mismo. También se pueden construir gráficos CUSUM y EWMA multivariantes. Un ejemplo de aplicación de éstos últimos puede encontrarse en Alfaro Navarro (2002).

En la Figura 6, está representado el gráfico multivariante que controla las dos características citadas. Se puede observar que existe una muestra fuera de control, en consecuencia habrá que buscar si existe alguna causa asignable investigando qué variable origina dicha situación. Uno de los procedimientos para averiguarlo es analizar cada variable individualmente. Observando el gráfico obtenido para la "Pilosidad" (Figura 4), podemos apreciar que la misma muestra número 11, está también fuera de control, luego el análisis de los datos parece indicar que es la "Pilosidad" la característica que está provocando el problema. Eliminando las muestras correspondientes y recalculando los límites, se obtiene el gráfico de la Figura 7. En él se tienen controladas conjuntamente las dos características y aporta los límites definitivos para la futura inspección. 
Figura 6. Gráfico multivariante. "Título absoluto" y "Pilosidad"

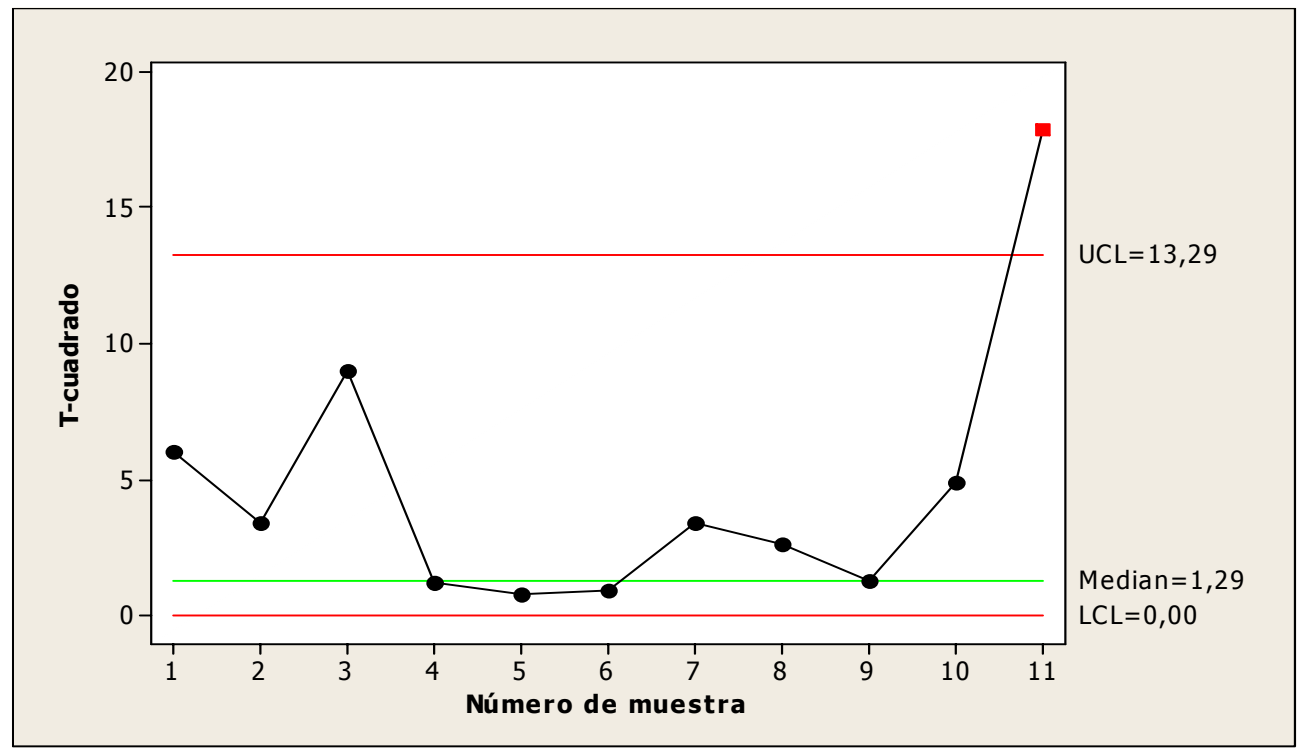

Figura 7. Gráfico multivariante. "Título absoluto" y "Pilosidad" (eliminando una muestra)

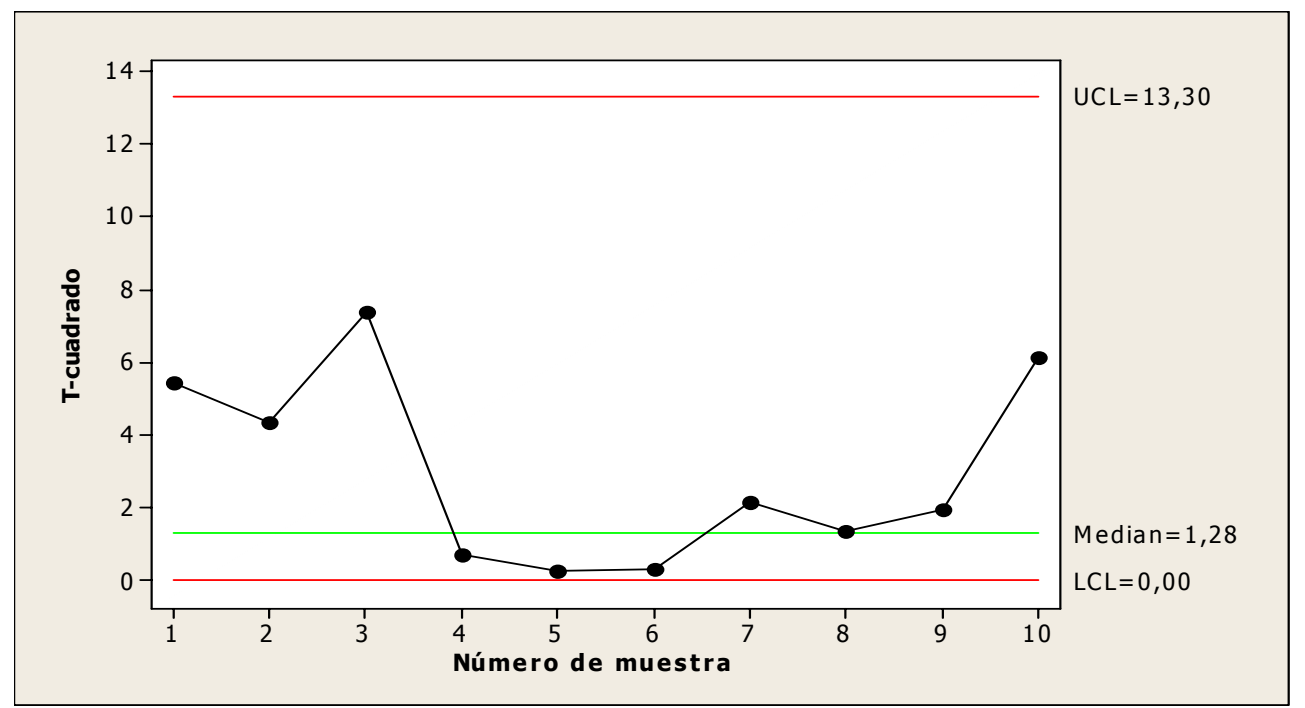

Curva Característica de Operación (CO)

Hasta ahora hemos considerado un tamaño de muestra de ocho unidades, puesto que es el que consideraba la empresa textil. Sin embargo, el tamaño muestral depende de los cambios que se quieran 
detectar. Una herramienta para elegir $n$ es la Curva CO. La Tabla 3 y la Figura 8 recogen los valores de $\beta$ para distintos cambios $k$ en la media del proceso medidos en unidades de desviación típica. Se compara dicha curva para tamaño de muestra $n=8$ y para $n=5$ cuando la media cambia de $\mu_{0}$ a $\mu_{1}$, con $\mu_{1}=\mu_{0}+k \sigma$.

Tabla 3. Valores de $\beta$ según tamaño de muestra

\begin{tabular}{|l|c|c|c|r|}
\hline \multirow{3}{*}{$\boldsymbol{k}$} & \multicolumn{2}{|c|}{$\boldsymbol{n}=\mathbf{8}$} & \multicolumn{2}{c|}{$\boldsymbol{n}=\mathbf{5}$} \\
\cline { 2 - 5 } & $\beta$ & $\frac{1}{1-\beta}$ & $\beta$ & $\frac{1}{1-\beta}$ \\
\hline 0 & 0,9973 & 370,379 & 0,9973 & 370,379 \\
\hline 0,2 & 0,9924 & 130,864 & 0,9944 & 177,730 \\
\hline 0,4 & 0,9691 & 32,409 & 0,9823 & 56,593 \\
\hline 0,6 & 0,9037 & 10,384 & 0,9514 & 20,564 \\
\hline 0,8 & 0,7695 & 4,339 & 0,8871 & 8,856 \\
\hline 1 & 0,5681 & 2,315 & 0,7775 & 4,495 \\
\hline 1,2 & 0,3467 & 1,531 & 0,6243 & 2,661 \\
\hline 1,4 & 0,1686 & 1,203 & 0,4481 & 1,812 \\
\hline 1,6 & 0,0636 & 1,068 & 0,2817 & 1,392 \\
\hline 1,8 & 0,0183 & 1,019 & 0,1527 & 1,180 \\
\hline 2 & 0,0039 & 1,004 & 0,0705 & 1,076 \\
\hline 2,2 & 0,0006 & 1,001 & 0,0275 & 1,028 \\
\hline
\end{tabular}

Figura 8. Curva CO

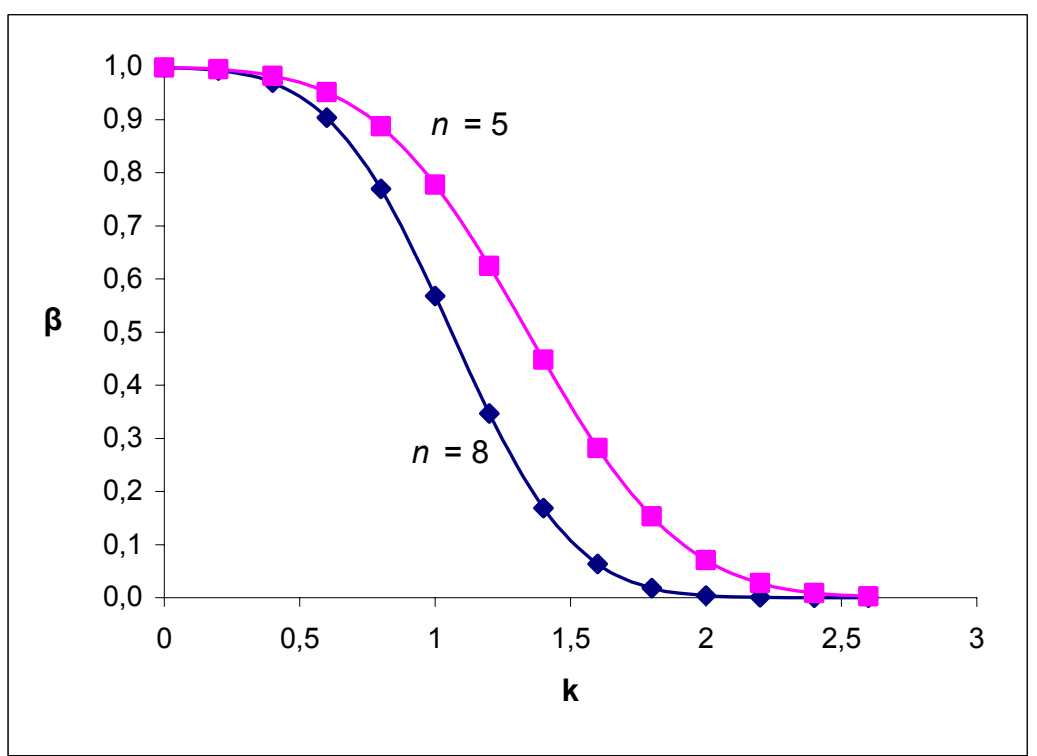


Como ya hemos señalado, $\beta$ representa la probabilidad de no detectar un cambio en la media del proceso (y por tanto de no rechazar $H_{0}$ ) cuando dicho cambio se ha producido ( $H_{0}$ es falsa). Evidentemente $1-\beta$ es la probabilidad de detectarlo.

A medida que aumenta el cambio (el valor de $k$ aumenta) el riesgo $\beta$ disminuye $y$, por tanto, es mayor la probabilidad de que el gráfico $\bar{X}$ lo detecte en la primera muestra después de producirse.

Además, se observa que, para tamaño de muestra $n=8, \beta$ siempre es menor que para $n=5$. En consecuencia con tamaño de muestra más pequeño $\beta$ es más elevado, siendo menos probable detectar los cambios en la primera muestra una vez que se ha producido.

El número medio de muestras necesario antes de detectar un determinado cambio es $1 /(1-\beta)$. Así, para un cambio en la media del proceso de $k=0,8$ unidades de desviación típica, el número medio de muestras es aproximadamente 4 con $n=8$ y aproximadamente 9 con $n=5$. Es decir, cuando $n$ disminuye, $1 /(1-\beta)$ aumenta.

\section{CONCLUSIONES}

El objetivo del presente trabajo se ha centrado en comprobar la utilidad de las técnicas estadísticas de control de procesos en el sector textil, estudiando características de calidad de la hilatura tanto de forma individual como conjunta.

En primer lugar se han presentado los fundamentos estadísticos elementales de los gráficos de control, en concreto, los gráficos para controlar la media y la dispersión de una característica de calidad y los gráficos multivariantes. A continuación se han aplicado en la evaluación de características de calidad de un proceso de hilatura textil.

Hay que señalar que los datos de partida se han obtenido directamente de los archivos existentes en el laboratorio de una empresa textil. La información con la que se ha trabajado y el método utilizado en su recolección condiciona la elección del gráfico adecuado y su aplicación.

Las características de interés, "Título absoluto" y "Pilosidad", se han estudiado individualmente mediante los gráficos $\bar{X}-R$, ya que dichas características son medibles numéricamente y la recogida de datos se efectúa en muestras de tamaño 8 . Para el estudio conjunto se ha recurrido a los gráficos multivarantes $T^{2}$ de Hotelling. 
En el caso concreto de la máquina analizada y en el periodo temporal considerado, los gráficos obtenidos correspondientes al periodo de vigilancia del proceso, muestran que el "Título absoluto" se encuentra bajo control, tanto para la media como para la dispersión, estimando una media para el grosor del hilo de 18,322.

Por lo que respecta a la característica "Pilosidad" fue necesario eliminar una de las muestras iniciales para conseguir mantener el proceso bajo control. La misma muestra originó una señal de fuera de control en el gráfico multivariante.

Todos los gráficos y límites definitivos obtenidos para cada variable podrían tomarse para vigilar la producción futura. Esto significa que a partir de ellos cada vez que se tome una muestra se representaría en el gráfico. Se debe tener en cuenta que los gráficos diseñados únicamente sirven para analizar la producción futura de la máquina para la cual han sido diseñados, es decir, para cada máquina y cada característica se han de construir los gráficos de control correspondientes.

Ahora bien, cuando se cumplan las hipótesis exigidas, los gráficos de control son una herramienta muy útil para detectar irregularidades y localizar las causas que originan los problemas.

\section{BIBLIOGRAFÍA}

Alfaro NAVARRO, J.L. (2002) "Métodos multivariantes en control estadístico de la calidad", Documentos de Trabajo. Facultad de Ciencias Económicas y Empresariales de Albacete. Universidad de Castilla La Mancha.

APARISI F.; Charles W. Champ (2004) "Double Sampling Hotelling's T ${ }^{2}$ Charts", XXVIII Congreso Nacional de Estadística e Investigación Operativa, Cádiz.

BANKS, J. (1998) Control de calidad. México: Ed. Limusa.

CALCUTT, R. (1995) "The rights and wrongs of control charts", Applied Statistics, 44,3,pp. 279-288.

CHENG, P.; DAWSON, S. (1998) "A study of statistical process control: practice, problems and training needs", Total Quality Management, 9, 1, pp. 3-20.

Clemente Marín, G.; Miró y Martínez, P.; SAnChos Rico, V.J. (2001) "Análisis del comportamiento diferenciado en máquinas similares de tejeduría". XXVI Congreso Nacional de Estadística e Investigación Operativa, Úbeda. 
DUNCAN, A.J. (1986) Quality control and industrial statistics (5th ed.). Homewood, Illinois: Ed. Irwin, R.D. Inc.

FUCHS, C.; KENETT, R.S. (1998) Multivariate Quality Control. New York. Ed. Marcel Dekker, Inc.

GARVIN. D.A. (1984) "What does product quality really mean?". Sloan Management Review, 26, 1, pp. 25-43.

GRANT, E.L.; LEAVENWORTH, R.S. (1988) Statistical quality control (6th ed.). New York: Ed. McGraw-Hill, Inc.

Grima, P.; TORT-MARTORELL, J. (1995) Técnicas para la gestión de la calidad. Madrid: Ed. Díaz de Santos.

JURAN, J.M. (1997) "Early SQC: A historical supplement", Quality Progress, September, pp. 73-81.

JURAN, J.M.; GRYNA, F.M. (1988) Quality control handbook (4th ed.). New York: Ed. McGraw-Hill.

Lee IVester, A.; Neefus, J.D. [ref de 22 de julio 2004] Industria de productos textiles en Enciclopedia de salud y seguridad en el trabajo. <http://www.mtas.es/Publica/enciclo/general/contenido/tomo3/89p df $>$

Miró I Martínez, P.; CAROt Sierra, J.M.; MARTínez Gómez, M.; Jabaloyes VIVAS, J.M. (2004) "Diseño y mejora de textiles técnicos mediante experimentos de mezclas", XXVIII Congreso Nacional de Estadística e Investigación Operativa, Cádiz.

MONTGOMERY, D.C. (1991a) Introduction to Statistical Quality Control (2th ed.). New York: Ed. John Wiley \& Sons, Inc.

Navarrete, E. (1998) Control Estadístico de la Calidad. Granada: Ed. Adhara.

OAKLAND, J.S. (1999) Statistical Process Control (4th ed.). Oxford: Ed. BH.

SHEWART, W.A. (1931; rpt.1980) Economic Control of Quality of Manufactured Product. Milwaukee, Wis.: Ed. ASQC Quality Press.

Ziemer, T.L. (1993) "Graphical Methods for Process Control", Handbook of Satatistic, 9. C.R. Rao, ed.; pp. 999-1019. 